\title{
Subdivision Algorithms and the Kernel of a Polyhedron*
}

\author{
R. J. Gardner ${ }^{1}$ and M. Kallay ${ }^{2}$ \\ ${ }^{1}$ Department of Mathematics, Western Washington University, \\ Bellingham, WA 98225-9063, USA \\ ${ }^{2}$ Electronic Data Systems Corporation, C4 Technology West, \\ Suite 300,13555 SE 36 th Street, Bellevue, WA 98006 , USA
}

\begin{abstract}
The kernel $K$ of a convex polyhedron $P_{0}$, as defined by $L$. Fejes Tóth, is the limit of the sequence $\left(P_{n}\right)$, where $P_{n}$ is the convex hull of the midpoints of the edges of $P_{n-1}$. The boundary $\partial K$ of the convex body $K$ is investigated. It is shown that $\partial K$ contains no two-dimensional faces and that $\partial K$ need not belong to $C^{2}$. The connection with similar algorithms from CAD (computer aided design) is explained and utilized.
\end{abstract}

\section{Introduction}

Suppose $P_{0}$ is a planar convex polygon. A sequence $\left(P_{n}\right)$ of polygons can be defined by letting $P_{n}$ be the midpoint polygon of $P_{n-1}$; that is, the convex hull of the midpoints of all the edges of $P_{n-1}$.

It can be shown $\lim _{n} P_{n}=\left\{c_{0}\right\}$, where $c_{0}$ is the centroid of $P_{0}$ (and of all the polygons $P_{n}$ ). Much more interesting is the beautiful result that the sequence $\left\{\alpha_{2 n} P_{2 n}\right\}$, where $\alpha_{2 n}$ are suitable scaling factors, converges to an affinely regular polygon. (This theorem, attributed in [9] to Neumann, was actually discovered earlier by Darboux [5]; it has been rediscovered many times (see p. 322 of [9]).) Moreover, apart from its aesthetic appeal, there is an important application, since the result is a central ingredient in the solution of Hammer's X-ray problem [10].

Several generalizations of the midpoint process in $\mathbf{R}^{2}$ have been studied (e.g., [2] and [12]), but here we are concerned with the three-dimensional analogue proposed by Fejes Tóth in [13]. If $P_{0}$ is a convex polyhedron in $\mathbf{R}^{3}, P_{n}$ is defined exactly as above. In this case, however, $\lim _{n} P_{n}=K\left(P_{0}\right)$, where $K$ is a convex body

\footnotetext{
* R. J. Gardner was supported in part by a von Humboldt fellowship.
} 
in $\mathbf{R}^{3}$ called the kernel of $P_{0}$. To see this, observe that since the midpoint algorithm in $\mathbf{R}^{2}$ preserves centroids, the set of all centroids of facets of the polyhedra $P_{n}$ lies in the boundary $\partial K$ of $K$. As a preliminary example, the reader may check that if $P_{0}$ is a regular tetrahedron, then $P_{1}$ is a regular octahedron, and $P_{2}$ is a cuboctahedron; and if $P_{0}$ is a cube, $P_{1}$ is again a cuboctahedron, yielding the interesting fact that all these polyhedra have the same kernel (up to homothety).

Fejes Tóth asked what can be said about the structure of $\partial K$. As far as we know, nothing is yet known. From examples the impression that, as $n$ increases, the size of the facets of $P_{n}$ should decrease (and so their number should be unbounded), is obtained. We confirm this in Theorem 4, and deduce (Corollary 6 ) that $\partial K$ contains no facets (two-dimensional faces). We also show that $\partial K$ need not belong to $C^{2}$ (Theorem 8). The obvious questions, whether $\partial K$ is strictly convex or smooth, remain unanswered (the latter was also posed by Fejes Tóth in [13]). However, our results are evidence for the intriguing conjecture, suggested by Zamfirescu, that the kernel is in some sense a "typical" convex body. Indeed, Klee showed that most convex bodies, in the sense of Baire category, are strictly convex and have a smooth boundary, and Gruber proved that most do not have a boundary belonging to $C^{2}$ (see [14] and the references given there).

We have a second motivation in this note, namely, to draw the attention of mathematicians to the connection between Fejes Toth's problem and some work in CAD (computer aided design). In CAD, processes called subdivision algorithms are sometimes used to model curves or surfaces. It has been found efficient to store a finite set of points, edges, and facets, and then generate new ones by some algorithm, formulated so that successive iterations converge to a limiting curve or surface, preferably smooth. Several such algorithms have been suggested, and in some of these the midpoint operation or a similar one plays a role.

We discuss two of these algorithms in Section 4, and apply one of them to Fejes Tóth's problem. This is a planar algorithm which was, in fact, considered by de Rham long before CAD existed. Its analysis is quite complicated, and de Rham devoted several papers to it. The proof of Theorem 8 of Section 4 indicates that the midpoint operation in three dimensions is actually analogous to de Rham's algorithm, rather than to the planar midpoint algorithm. Furthermore, the convex hull operation in three dimensions can create new edges in a way that is not easy to foresee. All this suggests that a full solution of Zamfirescu's conjecture may be difficult to obtain.

We thank David Levin for a careful reading of the manuscript which revealed a flaw in our previous version of Theorem 8 .

\section{Definitions}

If $E$ is a set, we denote by $d(E), \partial E$, int $E$, and relint $E$, the diameter, boundary, interior, and relative interior of $\mathrm{E}$, respectively.

Suppose $P$ is an $m$-dimensional convex polytope in $\mathbf{R}^{n}$. Let $M(P)$ be the midpoint polytope of $P$, i.e., the $m$-dimensional polytope in $\mathbf{R}^{n}$ formed by taking the convex 
hull of the midpoints of the edges of $P$. We write $M^{n}(P)$ for $M\left(M^{n-1}(P)\right), n=2$, $3, \ldots$, where $M^{1}(P)=M(P)$.

If $P_{0}$ is a convex polyhedron in $\mathbf{R}^{3}$, let $P_{n}=M\left(P_{n-1}\right)$ for $n=1,2, \ldots$ Write $K=\lim _{n} P_{n}$. Then $K=K\left(P_{0}\right)$ is called the kernel of $P_{0}$ (see [13]).

If $K$ is the kernel of $P_{0}$, we define the centroid set $C(K)$ of $K$ by $C(K)=$ $\left\{x: x\right.$ is a centroid of a facet of some $\left.P_{n}, n \in N\right\}$.

Suppose $F$ is a facet, i.e., a two-dimensional face, of the polyhedron $P_{n}$. If there is a facet $G$ of $P_{n-1}$ such that $F=M(G)$, we say that $F$ is of type 1 . Now suppose $F$ is a facet of $P_{n}$ which has the following property: there is a vertex $v$ of $P_{n-1}$ such that each vertex $w$ of $F$ is the midpoint of some edge of $P_{n-1}$ with $v$ as one endpoint. Then we call $F$ a type 2 facet of $P_{n}$. For each $n$, we let

$$
\delta_{n}=\max \left\{d(F): F \text { is a facet of } P_{n}\right\}
$$

and

$$
\delta_{n}^{(2)}=\max \left\{d(F): F \text { is a type } 2 \text { facet of } P_{n}\right\}
$$

As is usual, we denote by $C^{k}$ the class of curves (or surfaces) whose every point has a relative neighborhood which is the range of an open set in $\mathbf{R}$ (or $\mathbf{R}^{2}$, respectively) under a $k$-times continuously differentiable mapping. Smooth means belonging to $C^{1}$.

\section{The Kernel Has No Facets}

Lemma 1. For each $n$, every facet of $P_{n}$ is either of type 1 or of type 2.

Proof. Let $F$ be a facet of $P_{n}$. Let $\operatorname{proj}_{\pi}$ denote the orthogonal projection onto a plane $\Pi$ orthogonal to $F$, and let $h$ be the line in $\Pi$ containing $\operatorname{proj}_{\pi} F$.

If $\operatorname{proj}_{\pi} P_{n-1}$ lies on one side of $h$, it is easy to see that $F$ must be a type 1 facet of $P_{n}$. Suppose, then, that $h \cap \operatorname{int}\left(\operatorname{proj}_{n} P_{n-1}\right) \neq \varnothing$. By our choice of $\Pi, \operatorname{proj}_{\pi} P_{n}$ lies on one side of $h$. Let $E$ be the open half-plane in $\Pi$ on the other side of $h$. If two or more vertices of $P_{n-1}$ project into $E$, there is an edge of $P_{n-1}$ whose midpoint projects into $E$, contradicting $E \cap \operatorname{proj}_{n} P_{n}=\varnothing$. Therefore a unique vertex $v$ of $P_{n-1}$ projects into $E$. It follows that each vertex of $F$ must be the midpoint of an edge of $P_{n-1}$ which has $v$ as one endpoint, and so $F$ is of type 2.

Lemma 2. For each $n, \delta_{n}<\delta_{n-1}$.

Proof. If $F$ is a facet of $P_{n}$ of type 1 , then $F=M(G)$ for some facet $G$ of $P_{n-1}$. The diameter $d(G)$ is the distance between some pair of vertices of $G$, and $F$ contains none of these vertices. Therefore $d(F)<d(G) \leq \delta_{n-1}$. 
Suppose $F$ is a type 2 facet of $P_{n}$. Let $v$ be the vertex of $P_{n-1}$ from the definition. Let $\left\{w_{i}\right\}$ be the vertices of $F$ and, for each $i$, let $e_{i}$ be the edge of $P_{n-1}$ containing $v$ and $w_{i}$. Finally, let $G_{i}$ be any facet of $P_{n-1}$ containing $e_{i}$. Then

$$
\begin{aligned}
d(F) & <\frac{1}{2} \max _{i, j}\left\{d\left(e_{i}\right)+d\left(e_{j}\right)\right\} \\
& \leq \frac{1}{2} \max _{i, j}\left\{d\left(G_{i}\right)+d\left(G_{j}\right)\right\} \\
& \leq \frac{1}{2} \cdot 2 \delta_{n-1}=\delta_{n-1},
\end{aligned}
$$

and the lemma follows.

Remark 3. We do not know if there is a $c<1$, depending on $P_{0}$, such that $\delta_{n}<c \delta_{n-1}$ for each $n$. On the other hand, given $c<1$, there is a $P_{0}$ with $\delta_{1}>c \delta_{0}$. To see this, let $H$ be a regular hexagon in the $x y$-plane centered at the origin, and for $\varepsilon>0$ let $P_{0}(\varepsilon)=\operatorname{conv}\{(0,0, \pm \varepsilon), H\}$, i.e., a regular double pyramid over $H$. Then, given $c<1$, we can choose $\varepsilon$ small enough so that for $P_{0}(\varepsilon)$ we have $\delta_{1}^{(2)}>c \delta_{0}$. More elaborate examples show that, given $c<1$ and $n \geq 1$, there is a $P_{0}$ with $\delta_{n}^{(2)}>c \delta_{0}$.

Theorem 4. $\delta_{n} \rightarrow 0$.

Proof. Suppose the sequence $\left(\delta_{n}\right)$ does not converge to zero. Since $\delta_{n}<\delta_{n-1}$ by Lemma 2, we have $\delta_{n} \rightarrow a>0$ and $\delta_{n}>a$ for all $n$. Therefore there are sequences $\left(u_{n}^{(1)}\right)$ and $\left(u_{n}^{(2)}\right)$, where $u_{n}^{(1)}$ and $u_{n}^{(2)}$ are vertices of the same facet $F_{n}$ of $P_{n}$, such that $\delta_{n}=d\left(F_{n}\right)=\left\|u_{n}^{(1)}-u_{n}^{(2)}\right\| \rightarrow a$.

Suppose there is an $n_{0}$ such that $F_{n}$ is of type 1 for $n \geq n_{0}$. For each $n \geq n_{0}$, there is a least integer $k_{n}$ such that there are facets $G_{i}^{(n)}$ of $P_{i}$ with $F_{n}=G_{n}^{(n)}$ and $G_{i+1}^{(n)}=M\left(G_{i}^{(n)}\right), k_{n} \leq i \leq n-1$. Suppose there is an $m$ such that $k_{n} \leq m$ for all $n \geq n_{0}$. Then each $F_{n}, n \geq n_{0}$, is descended from some facet of some $P_{i}, 0 \leq i \leq m$, via the midpoint operation. However, there are only finitely many such facets, and for each one successive midpoint polygons converge to its centroid. Therefore, for sufficiently large $n$, we must have $d\left(F_{n}\right)<a$, which contradicts $\delta_{n}>a$. Thus $k_{n} \rightarrow \infty$ as $n \rightarrow \infty$. Let $F_{n}^{\prime}=G_{k_{n}}^{(n)}$. Then $F_{n}^{\prime}$ is not of type 1 , so by Lemma 1 it must be of type 2. Also, $d\left(F_{n}\right)<d\left(F_{n}^{\prime}\right) \leq \delta_{k_{n}}$, so $d\left(F_{n}^{\prime}\right) \rightarrow a$.

From the above it follows that we can assume there is a subsequence $S$ of $\mathbf{N}$, such that $F_{n}$ is of type 2 for $n \in S$ and $d\left(F_{n}\right) \rightarrow a$ as $n \rightarrow \infty$ through $S$. Choose a subsequence $T$ of $S$ such that the line segments $s_{n}=\left[u_{n}^{(1)}, u_{n}^{(2)}\right]$ converge in the Hausdorff metric to a line segment $s=\left[u^{(1)}, u^{(2)}\right]$ of length $a$ in $\partial K$, where $K$ is the kernel of $P_{0}$, as $n \rightarrow \infty$ through $T$. Let $t$ be the line containing $s$.

For each $n \in T$, there are vertices $v_{n}^{(i)}, 1 \leq i \leq 3$, of $P_{n-1}$ such that $u_{n}^{(i)}=$ $\frac{1}{2}\left(v_{n}^{(i)}+v_{n}^{(i+1)}\right)$, and $e_{n}^{(i)}=\left[v_{n}^{(i)}, v_{n}^{(i+1)}\right]$ is an edge of $P_{n-1}, i=1,2$. By taking a further subsequence if necessary, we may suppose that $e_{n}^{(1)}$ is the longer of these edges, i.e., $d\left(e_{n}^{(2)}\right)=c_{n} d\left(e_{n}^{(1)}\right)$ for some $c_{n} \leq 1$. Let $9_{n}$ be the angle between $e_{n}^{(1)}$ and $e_{n}^{(2)}$. Then

$$
\left[d\left(F_{n}\right)\right]^{2}=\frac{1}{4}\left(\left[d\left(e_{n}^{(1)}\right)\right]^{2}+\left[d\left(e_{n}^{(2)}\right)\right]^{2}-2 d\left(e_{n}^{(1)}\right) d\left(e_{n}^{(2)}\right) \cos \vartheta_{n}\right)
$$


giving $d\left(e_{n}^{(1)}\right)=\alpha_{n} d\left(F_{n}\right)$, where

$$
\alpha_{n}=\frac{2}{\left(1+c_{n}^{2}-2 c_{n} \cos 9_{n}\right)^{1 / 2}} .
$$

Now $\alpha_{n} \geq 1$, so $d\left(F_{n}\right) \leq d\left(e_{n}^{(1)}\right) \leq \delta_{n-1}$ for $n \in T$. Thus $\alpha_{n} \rightarrow 1$, as $n \rightarrow \infty$ through $T$. This implies that $c_{n} \rightarrow 1$ and $9_{n} \rightarrow \pi$, as $n \rightarrow \infty$ through $T$. It follows that $v_{n}^{(i)} \rightarrow v^{(i)}$ as $n \rightarrow \infty$ through $T$, where $v^{(i)}$ lies in $t \cap \partial K, 1 \leq i \leq 3,\left\|v^{(1)}-v^{(2)}\right\|=$ $\left\|v^{(2)}-v^{(3)}\right\|=a$, and $v^{(2)}$ is the midpoint of $s$.

The edges $e_{n}^{(1)}$ of $P_{n-1}$ converge to the segment $\left[v^{(1)}, v^{(2)}\right]$ of length $a$. Each $e_{n}^{(1)}$ belongs to a facet of type 1 or type 2 . In either case there are vertices $w_{n}^{(i)}$ of $P_{n-2}$, $1 \leq i \leq 3$, such that $v_{n}^{(i)}=\frac{1}{2}\left(w_{n}^{(i)}+w_{n}^{(i+1)}\right)$, and $f_{n}^{(i)}=\left[w_{n}^{(i)}, w_{n}^{(i+1)}\right]$ is an edge of $P_{n-2}$, $i=1,2$. Now the argument above shows that $w_{n}^{(i)} \rightarrow w^{(i)}$, where $w^{(i)}$ lies in $t \cap \partial \hat{K}$, $1 \leq i \leq 3,\left\|w^{(1)}-w^{(2)}\right\|=\left\|w^{(2)}-w^{(3)}\right\|=a$, and $w^{(2)}=u^{(1)}$ is the midpoint of $\left[v^{(1)}, v^{(2)}\right]$. Hence $\left\|w^{(1)}-v^{(3)}\right\|=5 a / 2$.

Replacing $e_{n}^{(1)}$ by $f_{n}^{(1)}$ and repeating the argument yields a point $x^{(3)}$ in $t \cap \partial K$ with $\left\|w^{(1)}-x^{(3)}\right\|=3 a$, and so on. We deduce that $\partial K$ contains line segments of arbitrary length, which is impossible.

Corollary 5. The centroid set $C(K)$ is dense in $\partial K$.

Proof. Let $\varepsilon>0$ and $x \in \partial K$ be given. There is an $n_{0}$ such that, for each $n \geq n_{0}$, there is a point $x_{n} \in \partial P_{n}$ with $\left\|x-x_{n}\right\|<\varepsilon / 2$.

By Theorem 4 there is an $n_{1}>n_{0}$ such that $\delta_{n}<\varepsilon / 2$ for each $n \geq n_{1}$. If $x_{n} \in F_{n}$, where $F_{n}$ is a facet of $P_{n}$, and $c_{n}$ is the centroid of $F_{n}$, we have

$$
\begin{aligned}
\left\|x-c_{n}\right\| & \leq\left\|x-x_{n}\right\|+\left\|x_{n}-c_{n}\right\| \\
& \leq \frac{\varepsilon}{2}+\frac{\varepsilon}{2}=\varepsilon
\end{aligned}
$$

for $n \geq n_{1}$, as required.

Corollary 6. $\partial K$ contains no facets.

Proof. Suppose $D$ is a facet in $\partial K$. Then there is a $c \in C(K)$ with $c \in$ relint $D$, by Corollary 5. For some $n, c$ is the centroid of a facet $F_{n}$ of $P_{n}$. Then $D \subset F_{n}$. However, if $H$ is the plane containing $F_{n}$, then $P_{n} \cap H \rightarrow\{c\}$, a contradiction.

\section{Subdivision Algorithms}

We begin by describing a planar algorithm studied by de Rham, which we then apply to obtain information about the kernel of a polyhedron.

Let $Q_{0}$ be a planar convex polygon with vertices $q_{0}, \ldots, q_{n-1}$. (We always take the indices of $q_{i}$ modulo $n$.) On the edge $\left[q_{i}, q_{i+1}\right]$, consider the points $q_{2 i}^{\prime}$ and 
$q_{2 i+1}^{\prime}$ which divide the edge into three segments, $\left[q_{i}, q_{2 i}^{\prime}\right],\left[q_{2 i}^{\prime}, q_{2 i+1}^{\prime}\right]$, and $\left[q_{2 i+1}^{\prime}, q_{i+1}\right]$, in the proportions $\beta_{1}, \alpha$, and $\beta_{2}$, respectively, where $\beta_{1}+\alpha+\beta_{2}=1$. The points $q_{i}^{\prime}, i=0, \ldots, 2 n-1$, form the vertices of a polygon $Q_{1}$. The process may be repeated, with the same proportions, to obtain a sequence $\left(Q_{n}\right)$ of polygons. Then $Q=\lim _{n} Q_{n}$ is a convex body in $\mathbf{R}^{2}$.

Let $\gamma_{i}=\beta_{i} / \alpha, i=1,2$. De Rham's interest in the limiting curve $\partial Q$ stems from the surprising fact that, for all but a single pair of values of the parameters $\gamma_{i}, \partial Q$ has singular properties. In a series of papers (see, for example, [6]) he showed that if $\gamma_{i} \leq 1, i=1,2$ (which includes the case where each side of the polygon $Q_{n}$ is trisected), then $\partial Q$ is smooth, but if $\gamma_{i}>1, i=1,2$, then $\partial Q$ has no tangent at a countable dense subset of $\partial Q$; further, unless $\gamma_{1}=\gamma_{2}=\frac{1}{2}$, no subarc of $\partial Q$ belongs to $C^{2}$.

In contrast to this is the special case $\gamma_{1}=\gamma_{2}=\frac{1}{2}$. Here it can be shown (e.g., Section 2 of [3]) that $\partial Q$ is a continuously differentiable curve consisting of a finite union of parabolic arcs, each joining adjacent midpoints of the sides of the original polygon $Q_{0}$. This was also known to de Rham, but in the CAD literature it is called Chaikin's algorithm (see [4], [11], or [3]), since until recently it was not realized that de Rham has priority. We need the following result concerning this algorithm.

Lemma 7. Let $Q_{0}$ be a planar convex polygon with vertices $q_{i}, i=0, \ldots, n-1$, and let $Q$ be the limiting convex body obtained from $Q_{0}$ by Chaikin's algorithm. Then $\partial Q$ belongs to $C^{2}$ if and only if $\left[q_{i-1}, q_{i+2}\right]$ is parallel to $\left[q_{i}, q_{i+1}\right]$ for $i=0, \ldots, n-1$.

Proof. It is known (see p. 120 of [3]) that $\partial Q$ is a finite union of parabolic arcs given by

$$
p_{i}(t)=\left(\frac{1}{2} q_{i-1}-q_{i}+\frac{1}{2} q_{i+1}\right) t^{2}+\left(q_{i}-q_{i-1}\right) t+\frac{1}{2}\left(q_{i-1}+q_{i}\right)
$$

for $0 \leq t \leq 1, i=0, \ldots, n-1$. The arc $p_{i}(t), 0 \leq t \leq 1$, joins the midpoints $p_{i}(0)=$ $\frac{1}{2}\left(q_{i-1}+q_{i}\right)$ and $p_{i}(1)=\frac{1}{2}\left(q_{i}+q_{i+1}\right)$ of two adjacent sides of $Q_{0}$. Note that

$$
p_{i}^{\prime}(1)=q_{i+1}-q_{i}=p_{i+1}^{\prime}(0)
$$

$i=0, \ldots, n-1$, which shows that $\partial Q$ belongs to $C^{1}$. Also, $p_{i}^{\prime \prime}(t)$ is constant, and the curvature at $p_{i}(t)$ is the length of the vector

$$
\frac{p_{i}^{\prime}(t) \times p_{i}^{\prime \prime}}{\left|p_{i}^{\prime}(t)\right|^{3}}
$$

$i=0, \ldots, n-1$. Using $p_{i}^{\prime}(1)=p_{i+1}^{\prime}(0)$, we see that $\partial Q$ belongs to $C^{2}$ if and only if

$$
p_{i}^{\prime}(1) \times p_{i}^{\prime \prime}=p_{i}^{\prime}(1) \times p_{i+1}^{\prime \prime}
$$

or

$$
p_{i}^{\prime}(1) \times\left(p_{i+1}^{\prime \prime}-p_{i}^{\prime \prime}\right)=0
$$


$i=0, \ldots, n-1$. This is equivalent to the condition that $p_{i+1}^{\prime \prime}-p_{i}^{\prime \prime}$ is parallel to $p_{i}^{\prime}(1), i=0, \ldots, n-1$. Since

$$
\begin{aligned}
p_{i+1}^{\prime \prime}-p_{i}^{\prime \prime} & =\left(q_{i}-2 q_{i+1}+q_{i+2}\right)-\left(q_{i-1}-2 q_{i}+q_{i+1}\right) \\
& =\left(q_{i+2}-q_{i-1}\right)+3\left(q_{i}-q_{i+1}\right) \\
& =\left(q_{i+2}-q_{i-1}\right)-3 p_{i}^{\prime}(1)
\end{aligned}
$$

this reduces to the condition that $q_{i+2}-q_{i-1}$ is parallel to $q_{i+1}-q_{i}$, $i=0, \ldots, n-1$, as required.

Theorem 8. There exists a polyhedron $P_{0}$ such that the boundary of the kernel of $P_{0}$ does not belong to $C^{2}$.

Proof. Let $Q_{0}$ be an quadrilateral in the $x y$-plane in $\mathbf{R}^{3}$ which is not a parallelogram. To ease our description, we also assume that $Q_{0}$ is not a trapezium, though this is not actually necessary. Suppose $q_{i}, 0 \leq i \leq 3$, are the vertices of $Q_{0}$. The polyhedron $P_{0}$ is the right cylinder with vertices $\left(q_{i}, \pm 1\right), 0 \leq i \leq 3$. We need to follow Fejes Tóth's algorithm through several steps.

Let $R_{i}$ be the rectangular facet of $P_{0}$ with vertices $\left(q_{i}, \pm 1\right)$ and $\left(q_{i+1}, \pm 1\right)$. Note that each $P_{n}$ will be symmetric about the $x y$-plane. For our purposes, it will suffice to fix $i$ and consider at the $n$th stage of the algorithm the facet $M^{n}\left(R_{i}\right)$ of $P_{n}$ and the set $\mathscr{F}_{i}^{+}\left(P_{n}\right)$ of facets of $P_{n}$ which meet both $M^{n}\left(R_{i}\right)$ and the open half-space $\{(x, y, z): z>0\}$.

The facet $M\left(R_{i}\right)$ of $P_{1}$ is a parallelogram, and the set $\mathscr{F}_{i}^{+}\left(P_{1}\right)$ consists of the $n$-gon $M\left(Q_{0} \times\{1\}\right)$ and two triangles, $S_{i}$ and $S_{i+1}$, with one vertex at $q_{i}, q_{i+1}$, respectively (see Fig. 1). The facet $M^{2}\left(R_{i}\right)$ of $P_{2}$ is rectangular. Two rectangles, $R_{i}^{(1)}, R_{i}^{(2)}$, each of which share a vertical edge with $M^{2}\left(R_{i}\right)$, are contained in $\mathscr{F}_{i}^{+}\left(P_{2}\right)$. The latter also contains two triangles, $S_{i}^{(1)}$ and $S_{i}^{(2)}$, which have an edge in common

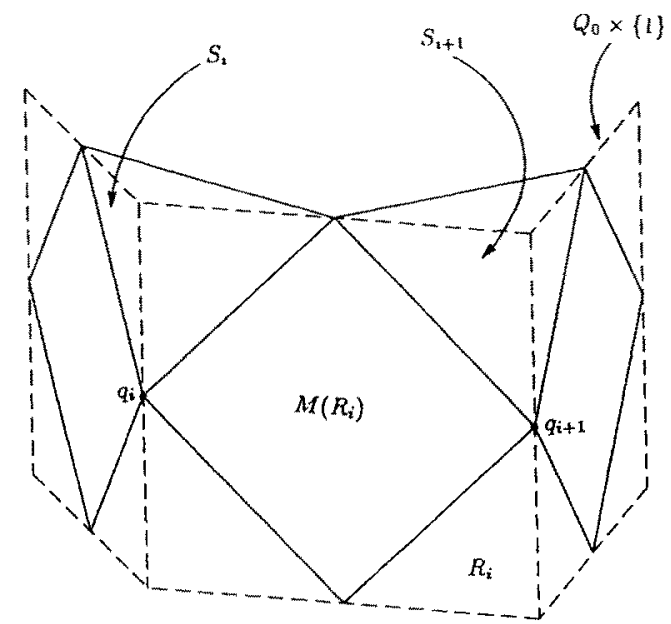

Fig. 1 


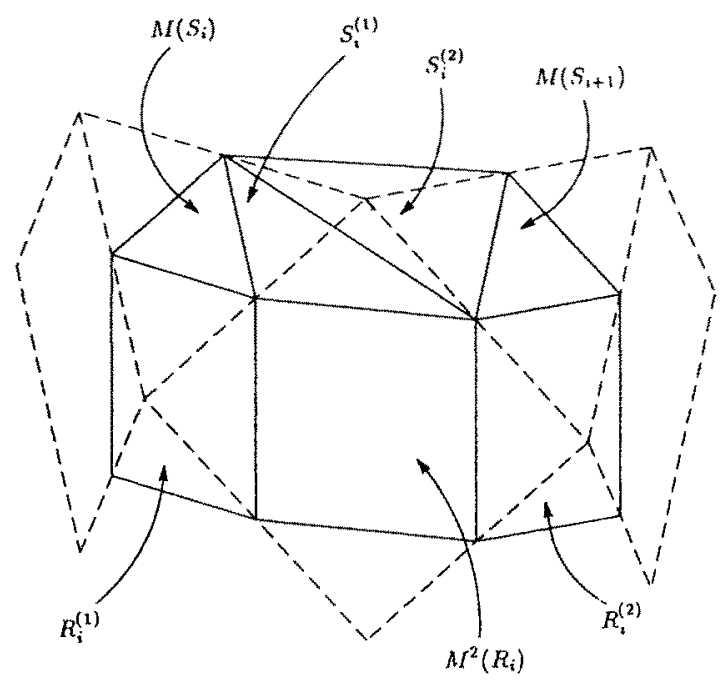

Fig. 2

with $M^{2}\left(R_{i}\right)$ and $M^{2}\left(Q_{0} \times\{1\}\right)$, respectively. Finally, $\mathscr{F}_{i}^{+}\left(P_{2}\right)$ contains the two triangles $M\left(S_{i}\right)$ and $M\left(S_{i+1}\right)$. We also assume without loss of generality that $S_{i}^{(1)}$ and $M\left(S_{i}\right)$ meet in an edge (see Fig. 2). The key observation here is that one edge of $M\left(S_{i}\right)$ is parallel to one diagonal of $M^{2}\left(R_{i}\right)$, and one edge of $M\left(S_{i+1}\right)$ is parallel to the other diagonal. This parallelism produces two trapezoidal facets at the next stage.

The following facets comprise $\mathscr{F}_{i}^{+}\left(P_{3}\right)$ (see Fig. 3). Firstly, the three parallelograms $M^{3}\left(R_{i}\right), M\left(R_{i}^{(1)}\right)$, and $M\left(R_{i}^{(2)}\right)$; two trapeziums, $T_{i}$ and $T_{i+1}$, which share an edge with $M^{3}\left(R_{i}\right)$ and with $M^{2}\left(S_{i}\right), M^{2}\left(S_{i+1}\right)$, respectively; a triangle $S_{i}^{\prime}$, which has

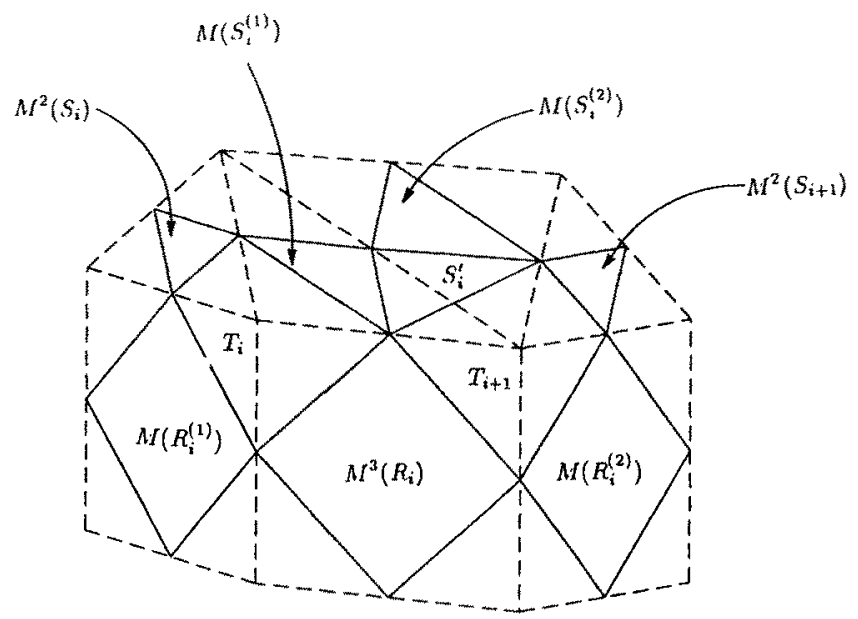

Fig. 3 


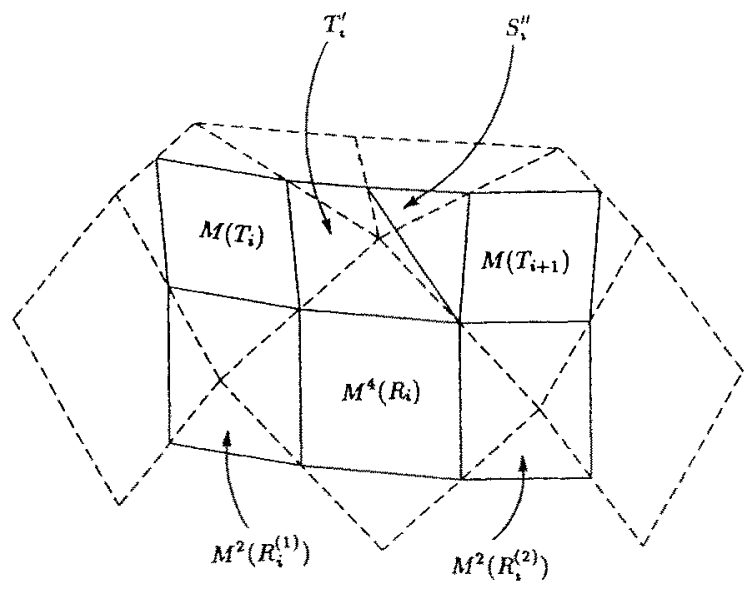

Fig. 4

common edges with $M\left(S_{i}^{(1)}\right), M\left(S_{i}^{(2)}\right)$, and $T_{i+1}$; and the triangle $M\left(S_{i}^{(1)}\right)$. Note that the latter has a horizontal edge, which is parallel to the horizontal diagonal of $M^{3}\left(R_{i}\right)$; this results in a trapezium at the next stage.

The next iteration produces the facets in $\mathscr{F}_{i}^{+}\left(P_{4}\right)$, which form a configuration (see Fig. 4) that is preserved by alternate steps of the algorithm. We have the rectangles $M^{4}\left(R_{i}\right), M^{2}\left(R_{i}^{(1)}\right)$, and $M^{2}\left(R_{i}^{(2)}\right)$; two parallelograms, $M\left(T_{i}\right)$ and $M\left(T_{i+1}\right)$; a trapezium $T_{i}^{\prime}$, which has common edges with $M^{4}\left(R_{i}\right)$ and $M\left(T_{i}\right)$; and a triangle $S_{i}^{\prime \prime}$, which meets both $T_{i}^{\prime}$ and $M\left(T_{i+1}\right)$ in an edge.

The parallelograms $M\left(T_{i}\right)$ and $M\left(T_{i+1}\right)$ each have a diagonal which is parallel to a diagonal of $M^{4}\left(R_{i}\right)$. Using this fact it is easy to see that $\mathscr{F}_{i}^{+}\left(P_{6}\right)$, and by induction, $\mathscr{F}_{i}^{+}\left(P_{2 n}\right)$, for $n \geq 3$, also contains three rectangles, two parallelograms, a trapezium, and a triangle, in the same configuration. In particular, the rectangles $M^{2 n}\left(R_{i}\right), M^{2 n-2}\left(R_{i}^{(1)}\right)$, and $M^{2 n-2}\left(R_{i}^{(2)}\right)$ are facets of $P_{2 n}$, and their centroids lie in the boundary of the kernel of $P_{0}$. The intersections of these rectangles with the $x y$-plane form three consecutive edges of the polygon $Q_{n}$ obtained from $Q_{0}$ by Chaikin's algorithm; namely, the edge whose midpoint is $\frac{1}{2}\left(q_{i}+q_{i+1}\right)$, together with the adjacent edge on either side. The midpoints of these edges, which are precisely the centroids of the just-mentioned rectangular facets of $P_{2 n}$, lie in the limiting curve $\partial Q$ obtained from $Q_{0}$ by Chaikin's algorithm.

It follows that the kernel $K\left(P_{0}\right)$ contains two sequences of points in the $x y$-plane, each converging to $\frac{1}{2}\left(q_{i}+q_{i+1}\right)$. One of these sequences belongs to the parabolic arc of $\partial Q$ with endpoints $\frac{1}{2}\left(q_{i-1}+q_{i}\right)$ and $\frac{1}{2}\left(q_{i}+q_{i+1}\right)$, and the other to the parabolic arc of $\partial Q$ with endpoints $\frac{1}{2}\left(q_{i}+q_{i+1}\right)$ and $\frac{1}{2}\left(q_{i+1}+q_{i+2}\right)$. Our assumptions on $Q_{0}$ imply, by Lemma 7 and its proof, that the intersection of the boundary of $K\left(P_{0}\right)$ with the $x y$-plane does not belong to $C^{2}$. Therefore the boundary of $K\left(P_{0}\right)$ itself is not in $C^{2}$.

Chaikin's algorithm-de Rham's with $\gamma_{1}=\gamma_{2}=\frac{1}{2}$ motivated several algorithms in $\mathbf{R}^{3}$. For example, the Doo algorithm in [7], following earlier work of 
Catmull and Clark, "smoothes" a polyhedron by an iteration very similar in spirit to Fejes Tóth's algorithm. For a convex polyhedron $P_{0}$, the Doo algorithm proceeds as follows. If $F$ is a facet of $P_{0}$, a new facet is formed by joining, by line segments, the midpoints of the line segments whose endpoints are the centroid of $F$ and a vertex of $F$. If $F$ is an $m$-gon, the new facet is also an $m$-gon, called a type $F$ facet. Suppose $F_{1}$ and $F_{2}$ are facets of $P_{0}$ with an edge $e$ in common. If $G_{1}$ and $G_{2}$ are the corresponding new type $F$ facets, then $G_{1}$ and $G_{2}$ each have an edge parallel to $e$ and of length half that of $e$. Joining the corresponding endpoints of these edges by line segments, we obtain a parallelogram. Such parallelograms will also be new facets, called type $E$ (for edge) facets. Finally, suppose $v$ is a vertex of $P_{0}$. For each facet $F$ of $P_{0}$ with $v$ as a vertex, there is a new type $F$ facet $G$ contained in $F$. Facet $G$ has a vertex $v_{F}$ at the midpoint of the line segment joining the centroid of $F$ with $v$. We consider the set of points $v_{F}$ for such $F$ to be the vertices of a new "facet," which however will not in general be planar. We call these new "facets" type $V$.

In this way a new "polyhedron" $P_{1}$ is defined. We now iterate this construction-using all facets, planar or not--to obtain a sequence $\left(P_{n}\right)$ of "polyhedra." The limiting body $H$ we call the Doo kernel of $P_{0}$.

Although this algorithm is more complicated to describe than Fejes Tóth's, the analysis of $\partial H$ is in some ways easier, since its construction does not employ the rather unpredictable convex hull operation. For example, the type $F$ and type $V$ facets at each stage are surrounded by a carpet of type E facets (parallelograms). In the CAD literature some efforts have been made to determine the smoothness properties of $\partial H$ and the boundaries of kernels of other algorithms (see [1], [7], [8], and Section 4 of [3]). Our final result, however, shows that the Doo kernel suffers from the disadvantage that it does not preserve convexity.

Theorem 9. There is a convex polyhedron whose Doo kernel is not convex.

Proof. Let $P_{0}$ be a convex polyhedron with the following properties:

(i) $P_{0} \cap\{(x, y, z): z \geq 0\}$ is the pyramid with vertices $(0,0,1),( \pm 1,0,0)$, and $(0, \pm 1,0)$.

(ii) The segments $[(0,1,0),(1,0,0)]$ and $[(0,-1,0),(-1,0,0)]$ are edges, so that there are two triangular facets, $T_{1}$ and $T_{2}$, above the first and third quadrants in the $x y$-plane.

(iii) The two facets containing $(0,0,1)$ above the second and fourth quadrants are hexagons, $H_{1}$ and $H_{2}$, which extend below the $x y$-plane and are such that $H_{2}$ is the reflection of $H_{1}$ in the $z$-axis.

(iv) The lowest facet of $P_{0}$ is horizontal and has its centroid, $b$ say, on the $z$-axis.

Suppose that $t_{i}$ is the centroid of $T_{i}$ and $h_{i}$ is the centroid of $H_{i}$ for $i=1,2$. The type V "facet" of $P_{1}$ corresponding to the vertex $v=(0,0,1)$ of $P_{0}$ has four vertices, at $\frac{1}{2}\left(t_{i}+v\right), \frac{1}{2}\left(h_{i}+v\right), i=1,2$. The centroid of this "facet" is $c=$ $(v / 2)+\left(t_{1}+t_{2}+h_{1}+h_{2}\right) / 8$. Our assumptions (i)-(iii) above imply that $c$ lies on the $z$-axis, and it is clear that $c \neq b$.

Now, for $i=1,2, t_{i}$ lies in the boundary $\partial H$ of the Doo kernel $H$ of $P_{0}$. If $H$ 
is convex, the point $p$ at the intersection of $\left[t_{1}, t_{2}\right]$ and the $z$-axis belongs to $H$ and has positive $z$-coordinate. However, both $b$ and $c$ belong to $\partial H$, and by choosing $H_{i}$ sufficiently long we may ensure that $c$ has a negative $z$-coordinate, as does $b$. This is impossible, so $H$ cannot be convex.

\section{References}

[1] A. A. Ball and D. I. T. Storry. Conditions for tangent plane continuity over recursively generated B-spline surfaces. ACM Trans. Graphics, 7:83-102, 1988.

[2] M. Bourdeau and S. Dubuc. Litération de Fejes Tóth sur un polygone. J. Geom., 6:65 75, 1975.

[3] A. S. Caveretta and C. A. Micchelli. The design of curves and surfaces by subdivision algorithms. In Mathematical Methods in Computer Aided Design, T. Lyche and L. L. Schumaker, eds., pp. 115-153. Academic Press, New York, 1989.

[4] G. M. Chaikin. An algorithm for high speed curve generation. Comput. Graphics Image Process., 3:346-349, 1974.

[5] M. G. Darboux. Sur un problème de géométrie élémentaire. Bull. Sci. Math., 2:298-304, 1878.

[6] G. de Rham. Sur les courbes limites de polygones obtenus par trisection. L'Enseign. Math., 5:729-747, 1959.

[7] D. W. H. Doo. A subdivision algorithm for smoothing down irregular shaped polyhedrons. In Proceedings: Interactive Techniques in Computer Aided Design, Bologna, 1978, pp. 157-165.

[8] D. Doo and M.Sabin. Behaviour of recursive division surfaces near extraordinary points. Comput. Aided Design, 10:356-360, 1978.

[9] J. C. Fischer, D. Ruoff, and J. Shilleto. Polygons and polynomials. In The Geometric Vein, C. Davis, B. Grünbaum, and F. A. Sherk, eds., pp. 321-333. Springer-Verlag, New York, 1981.

[10] R. J. Gardner and P. McMullen. On Hammer's X-ray problem. J. London Math. Soc., 21:171-175, 1980.

[11] R. F. Reisenfeld. On Chaikin's algorithm. Comput. Graphics.Image Process., 4:304-310, 1975.

[12] G. Fejes Tóth. Iteration processes leading to a regular polygon (in Hungarian). Mat. Lapok, 23:135-141, 1972. (Math. Rev., 49, \#3683.)

[13] L. Fejes Tóth. Sequences of polyhedra. Amer. Math. Monthly, 88:145-146, 1981.

[14] T. Zamfirescu. Nearly all convex surfaces are smooth and strictly convex. Monatsh. Math., 103:57-62, 1987.

Received December 14, 1990, and in revised form October 21, 1991. 